\title{
XVII.
}

Aus der chirurgischen Klinik des Prof. Dr. Lucke in Strassburg.

\section{Die Behandlung tuberculöser Knochen- und Gelenkerkrankungen mit Jodoform-Glycerin.}

\author{
Von \\ Dr. Brodnitz, \\ Assistenzarzt der Klinik.
}

Die Resultate der operativen Behandlung der tuberculösen Knochen- und Gelenkerkrankungen sind zweifelsobne seit Einfuhrung des Jodoforms in die Wundbehandlung bedentend bessere geworden. Ob

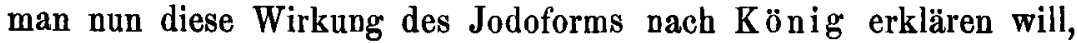
der unter dem Jodoform eine so reizlose und rasche Heilung eintreten sieht, dass Tuberculose in dem rasch schrumpfenden, vernarbenden Gewebe nicht zur Entwicklung kommt, oder nach Mosetig v. M o orh off, der eine specifische Wirkung annimmt, die Thatsache, dass das Jodoform die Heilung wesentlich unterstuitzt, bleibt bestehen.

Das Jodoform dürfte denn wohl auch ïberall bei Behandlung tuberculöser Processe Verwendung finden, und nur die Art und Weise der Anwendung ist eine verschiedene.

Wir selbst verwenden seit 3 Jabren das Jodoform nach Billroth's Empfehlung in Form einer 10 proc. Jodoform-Glycerinmischung, welche jedoch sterilisirt wird.

Obne hier des Näheren auf die von Ch. Heyn und Rokild Krosing aufgestellten Behauptungen, dass das Jodoform uiberhaupt kein Antisepticum sei, und die Untersuchungen de Reyter's, welcher dem Jodoform eine sehr bedeutende antiseptisehe Wirkung zuweist, wenn es mit Wundsecreten, resp. Gewebsflüssigkeiten bei Bluttemperatur in Verbindung tritt, einzugehen, sahen wir uns durch eigene Erfabrungen genöthigt, nur sterilisirtes Jodoform-Glycerin zu verwenden. 
Wir bekamen nämlich gleich im Anfange bei Verwendung des Jodoform-Glycerins nach einer Injection, welche unter allen aseptischen Cautelen ausgeführt war, ein Erysipel, welches vom Stichkanal ausging, 8 Stunden nach der Injection. Es wurden nunmehr von dem dem Flaschenhalse noch anhaftenden Jodoform-Glycerin Platten gegossen, und hierbei entwickelten sich so zahlreiche Bacteriencolonien, dass man von Keimfreiheit nicht sprechen konnte. Für uns erwuchs hieraus die Verpflichtung, nur sterilisirtes Jodoform-Glycerin fortan zu verwenden.

Die Sterilisation wird nach Angabe unseres damaligen Assistenten Dr. E. Levy und des Spitalapothekers Dr. Schneegans ${ }^{1}$ ), wie folgt, vorgenommen:

Es werden gewöhnliche Arzneigläser à 20,0 Grm. mit je 2,0 Grm. Jodoform und 18,0 Grm. Glycerin beschickt, gut verkorkt und die Korke mittelst Bindfadens befestigt. Die Fläschchen werden alsdann im Kochsalzbade einer Temperatur von $110^{\circ}$ während 2 bis 3 Stunden ausgesetzt, wonach sie mit Hülfe einer Zange aus dem heissen Bade herausgenommen und mit dem Kork bis zur Hälfte des Halses in geschmolzenes Paraffin eingetaucht werden. Dieses Letztere muss so schnell als möglich vorgenommen werden, um beim Abkühlen das Eindringen der Luft von aussen durch die Poren des Korks zu verhindern. Da beim Erkalten leicht kleine Risse in der Paraffinschicht auftreten, missen die Korke mehrmals überzogen werden.

Vor dem Gebrauch werden die Fläschchen in Sublimatlösung gelegt und alsdann in Schälchen, die ebenfalls in Sublimat gelegen haben, entleert.

Bei dieser Art der Sterilisation ist es uns möglich, stets eine grössere Menge sterilisirten Jodoform-Glycerins in kleineren Quantitäten vorräthig zu haben. Der Verlust bei etwaigem nicht vollstän. digen Verbrauch eines angebrochenen Fläschchens ist nur unbedeutend. In nur etwa 5 Proc. der so hergestellten Fläschchen entwickelt sich freies Jod, das man leicht an der braunrothen Färbung des Glycerins erkennt. Diese Fläschchen werden wegen der reizenden Wirkung des freien Jod von der Verwendung ausgeschlossen.

Wir verwandten nun das sterilisirte Jodoform-Glycerin entweder zu Injectionen, nachdem zuvor etwaige Abscesse entleert worden waren, oder wir verfuhren nach Angabe Billroth's, inden wir uns durch operativen Eingriff die erkrankten Theile zugänglich machten,

1) Journal de Pharmacie von Elsass-Lothringen. 18. Jahrgang. S. 9. Die Sterilisation des Jodoform-Glycerin von Dr. Schneegans, Spitalapotheke. 
das Erkrankte nach Möglichkeit zu entfernen suchten, die Wundhöhle mit Jodoform-Glycerin anfüllten und alsdann primär, resp. secundär die Naht legten, oder aber wir verzichteten nach dem operativen Eingriff völlig auf die Naht, tränkten sterilisirte Gazestreifen mit der Jodoform.Glycerinmischung und führten mit dieser sterilisirten Jodoform-Glyceringaze eine ständige Ausstopfung aus.

Nach diesen drei Methoden sind die angefuhrten 87 Fälle behandelt worden. Die im Laufe der letzten 6 Monate operativ behandelten Fälle sind nicht berilcksichtigt worden. Das definitive Resultat der erwähnten Fälle ist entweder durch eigenen Augenschein oder durch briefliche Anfrage festgestellt worden.

Von 5 Patienten mit kalten Abscessen, Fall 1-5 (ohne Gelenkbetheiligung), welche durch Punction und nachfolgende Injection von Jodoform-Glycerin behandelt worden sind, sind drei definitiv geheilt (Fall 1-3), bei zweien war schliesslich ein operativer Eingriff nöthig (Fall 4, 5).

Von 7 Patienten mit kalten Abscessen, Fall 4, 6, 7, 8, 9, 10, 11, welche durch Incision, Auslöffelung, nachfolgendes Anfüllen mit Jodoform-Glycerin und Primärnaht behandelt worden sind, sind 5 geheilt entlassen worden; von diesen 5 haben 3 zur Zeit Fisteln, einer ist nicht auffindbar, und nur einer ist als definitiv geheilt zu betrachten (Fall 4).

Von 4 Patienten mit kalten Abscessen, welche durch Incision und Auslöffelung und nachfolgendes Anfullen mit Jodoform-Glycerin und Secundärnaht behandelt worden sind (Fall 5, 12-14), sind 4 geheilt entlassen worden. Von diesen haben 2 zur Zeit Fisteln, einer ist gestorben, ohne dass etwas Näheres bekannt ist, und nur einer ist definitiv geheilt (Fall 12).

Von 7 Patienten mit kalten Abscessen, welche nach Incision und Auslöffelung mit ständiger Ausstopfung mit in Jodoform-Glycerinmischung getauchter Gaze behandelt worden sind (Fall 6, 15-20), ist einer ungeheilt, 3 sind mit kleiner Fistel in Heilung und 3 geheilt entlassen worden. Von diesen letzten 6 sind 4 definitiv geheilt (Fall $6,15,16,20)$, einer hat noch die Fistel, einer ist nicht auffindbar.

Wir haben also von den 20 Patienten mit kalten Abscessen über 3 nichts Näheres erfahren können, 9 sind definitiv als geheilt zu betrachten, 8 baben Fisteln. Von den 9 definitiv Geheilten sind 3 dureh Punction und nachfolgende Injection, 4 durch ständige Ausstopfung nach Incision, einer durch Incision mit Primärnaht, einer durch Incision mit Secundärnaht behandelt worden. 
Von 5 mit Punction und Injection Behandelten sind 3 definitiv geheilt.

Von 7 mit Incision und Ausstopfung Behandelten sind 4 definitiv geheilt.

Von 11 mit Incision und Primär- resp. Secundärnaht Behandelten sind nur 2 definitiv geheilt.

Von 6 Patienten, die an Rippencaries litten, und denen deshalb die Rippe resecirt worden ist (Fall 21-26), ist bei einem nach Anfulllung der Höhle mit Jodoform-Glycerin die Primärnaht angelegt worden, 5 sind mit ständiger Ausstopfung behandelt worden. Ersterer ist geheilt entlassen worden, hat zur Zeit jedoch eine Fistel; von den letzteren sind 2 ungeheilt, 3 sind geheilt entlassen worden (Fall 24, 25, 26).

Von 3 Patienten mit Herderkrankungen, ohne Gelenkbetheiligung (Fall 27-29), sind 3 nach der Incision und Auslöffelung mit ständiger Ausstopfung behandelt worden. Alle 3 sind geheilt entlassen worden und sind zur Zeit noch geheilt (Fall 27-29).

In 18 Fällen von ostaler Form der Gelenkerkrankung ohne Abscedirung (Fall $30-47$ ) ist nach der Resection und Anfüllen der Wundhöhle mit Jodoform-Glycerin in 8 Fällen die Primärnaht angelegt worden; alle 8 sind gebeilt entlassen worden; von diesen haben 2 Recidive, einer hat eine Fistel (Fall 33), aber keine Schmerzhaftigkeit, 5 sind definitiv geheilt (Fall 30, 34, 45, 46, 47). In 10 Fällen ist die Secundärnaht angelegt worden; alle 10 sind geheilt entlassen worden; einer ist 3 Monate später gestorben, 9 sind definitiv geheilt (Fall 36-44).

In 25 Fällen ostaler Form der Gelenkerkrankung, theils mit Fistel-, theils mit Abscessbildung (Fall 48-72), ist nach der Resection und Auslöffelung ständig mit Jodoform-Glycerin ausgestopft worden. Von diesen ist einer während der Behandlung gestorben, 4 sind ungeheilt und 20 geheilt entlassen worden; von diesen letzteren ist einer nicht auffindbar, 4 sind gestorben, 5 haben Recidive, und 10 sind definitiv geheilt (Fall 48, 51, 53, 55, 56, 60, 63, $67,70,72)$.

Von 9 Fällen von Kapseltuberculose (Fall 43, 73-80) ist nach der Arthrektomie und Anfüllung mit Jodoform-Glycerin in 7 Fällen die Primärnaht angelegt worden; alle 7 sind geheilt entlassen; einer bekam Recidiv (Fall 43), einer ist nicht auffindbar, 5 sind definitiv geheilt (Fall 73, 75, 76, 79, 80).

In 2 Fällen wurde die Secundärnaht angelegt; beide sind geheilt entlassen, beide definitiv geheilt (Fall 77, 78). 
Von 5 Fällen von Kapseltuberculose, die mit Injection von Jodoform-Glycerin behandelt worden sind (Fall 81-85), sind alle 5 geheilt; allerdings besteht nur bei 2 Fällen (Fall 81,82 ) die Heilung länger als $1 \mathrm{Jahr}$; bei den übrigen 3 ist die Zeit nach der letzten Injection zu kurz, um ein definitives Urtheil zu fällen. Von diesen 5 Fällen betreffen 2 das Fuss-, 2 das Knie- und einer das Handgelenk. In 2 Fällen von Hüftgelenkserkrankung mit Abscedirung, die mit Injection behandelt worden sind, ist in einern Fall Heilung erzielt worden (Fall 86); in dem zweiten war keine Besserung zu constatiren, so dass alsdann operativ vorgegangen werden musste (Fall 87).

Suchen wir nun aus den in Küze hier zusammengestellten Resultaten ein Urtheil über den Werth und die Zweckmässigkeit der drei verschiedenen Anwendungsarten des Jodoform-Glycerin zu gewinnen.

Die Injectionsmethode hat ihre grossen Vorzige und ist empfehlenswerth für die Fälle von Senkungsabscessen, bei denen sich durch die anatomischen Verhältnisse, Nähe der Körperhöhlen oder grossen Gefässe, die Ausräumung der Abscesshöhle nach Eröffnung durch Schnitt von selbst verbietet, sowie bei all' den Fällen, in denen wir entweder den eigentlichen Krankheitsherd nicht kennen oder nicht erreichen können; leider ist dieses ja bei den eigentlichen Senkungsabscessen nur zu bäufig der Fall. In diesen Fällen zu incidiren, die Abscesshöhle auszuräumen, dann mit Jodoform-Glycerin anzufüllen und zu nähen, ist nach unseren Erfahrungen durchaus unzweckmässig. Das Ziel, das uns bei dem operativen Eingriff vorschwebt, die Inangriffnahme des eigentlichen Krankheitsherdes, wird nicht erreicht, wir verschlechtern nur die äusseren Verhältnisse, indem wir statt der intacten Haut eine junge Narbe setzen. Der eigentliche Krankheitsherd, die Quelle des Eiters, besteht fort, und wenn wir auch vielleicht hoffen ditrfen, dass das in der Wundböhle befindliche Jodoform den Herd zur Ausheilung bringen wird, so sind wir zu der gleichen Hoffnung bei der Injection berechtigt. Die Entfernung des Eiters kann ja auch durch den Pottin erfolgen, und dass auf die Entfernung der Abscessmembran selbst nicht zu grosser Werth zu legen ist, haben die Untersuchungen ron Bruns und $\mathrm{Nau}$ werck gelelirt, welche aufs Klarste bewiesen haben, dass diese Membran sich unter dem Jodoform umzubilden im Stande ist. Der momentane Erfolg ist ja in diesen mit Incision behandelten Fällen ein glänzender; so wurden auch von unseren 11 so bebandelten Fällen 9 obne Fisteln entlassen; aber nur 2 blieben dauernd geheilt, bei den tibrigen bildete sich in der Narbe eine secernirende Fistel. 
Bei der Tuberculose der Gelenke haben wir in nur 5 Fällen von nicht sehr weit vorgeschrittener Kapselerkrankung die Injectionsmethode angewandt. In den weiter vorgeschrittenen Fällen jedoch, sowie bei der ostalen Form der Gelenkerkrankung, bei der die Patienten gar oft schon durch den Sitz des Leidens an das Bett gebunden sind, eine Behandlungsmethode einzuschlagen, bei der ganz ungewiss erst im Verlaufe von Wochen eine Besserung zu erwarten ist, halten wir uns nicht für berechtigt. - Die Resultate bei Behandlung jener 5 Fälle sind ermuthigend; zwei sind als völlig geheilt zu betrachten, da ein Jahr seit der letzten Injection verstrichen ist, ohne dass Recidive aufgetreten sind; die ubrigen drei sind ebenfalls geheilt, doch ist die Beobachtungszeit noch zu kurz, um ein definitives Urtheil zu fällen. Diese Resultate ermuthigen uns, auch in Zukunft bei Kapseltuberculose, die nicht zu weit vorgeschritten ist, diese Behandlungsmethode fortzusetzen; leider liefert uns unser klinisches Material selten so giinstige Fälle.

Für die ostale Form der Gelenkerkrankungen halten wir diese Behandlungsmethode fẗr nicht empfehlenswerth. Denn während wir bei der Kapseltuberculose darauf rechnen können, dass das injicirte Jodoform uberall gleichmässig auf die Granulationen zur Wirkung kommen kann, ist bei der ostalen Form die Hoffnung, jeden und alle Herde durch die Injection zu treffen und so unter die Jodoformwirkung zu setzen, doch wohl illusorisch. Aus der Weichbeit des Knochens beim Einstich der Cantlle einen Herd diagnosticiren zu wollen, wie es Schuller thut, durfte nicht immer richtig sein, da erfahrungsgemäss Osteoporose durchatts noch nicht mit tuberculöser Erkrankung des Knochens identisch ist.

In nur 2 Fällen von Hüftgelenkserkrankung ostaler Form mit Abscessbildung (Fall 86 u. 87) haben wir uns zur Injectionsbebandlung entschlossen. Bei dem einen Fall (Fall 86), der alle Zeichen der Coxitis darbot, war oberhalb des Trochanter deutlich in der Tiefe ein kleiner Abscess zu fühlen; die Basis des Scarpa'schen Dreiecks war zwar auf Druck empfindlich, zeigte jedoch keine deutliche Schwellung; die Bewegungen im Hüftgelenk waren beschränkt und schmerzhaft. Es wurde oberbalb des Trochanter nach dem Gelenk hin punktirt und nach Aspiration von Eiter mehrmals Jodoform-Glycerin injicirt; es trat völlige Heilung mit Ankylose im Hüftgelenk ein. In dem zweiten Falle (Fall 87), wo nicht nur oberhalb des Trochanter, sondern auch in den Adductoren, sowie an der Basis des Scarpa'schen Dreiecks deutliche Abscesse waren, trat trotz wiederholter Injection von JodoformGlycerin keine Besserung ein; bei der später vorgenommenen Operation 
fand sich ein Herd am äusseren Rande der Pfanne, ausserhalb der Kapsel, ein zweiter am inneren Pfannenrande, ferner ein dritter in der Pfanne selbst. Wenn in diesem Falle durch die Injection auf alle drei Herde eine Jodoformwirkung erzielt worden wäre, so wäre dieses doch wohl nur ein Zufall zu nennen. Im ersten Falle (Fall 81) handelte es sich wahrscheinlich überhaupt nur um eine Herderkrankung ausserhalb der Kapsel am Pfannenrande mit indirecter Betheiligung des Gelenkes. Es wutrde dieses ganz dem Fall Nr. 15 entsprechen; der Knabe kam mit fast rechtwinkliger Ankylose im Hüftgelenk und einem grossen, stark gespannten Abscess oberhalb des Trochanter in die Klinik. Der Abscess wurde nach der Incision durch ständige Ausstopfung mit Jodoform. Glyceringaze geheilt; er ging von einem Herd am äusseren Pfannenrande aus. Bei der später aus orthopädischen Grinden vorgenommenen Resection zeigte weder Kopf noch Pfanne irgend welche tuberculöse Erkrankung.

Dass das Bill roth'sche Verfahren, welches operatives Vorgehen, möglichste Entfernung alles Krankhaften, Ausfïllung der Wundhöhle mit Jodoform-Glycerin und Naht verlangt, an sich den Chirurgen sympathischer ist, bedarf wohl kaum der Erklärung. Bei diesem Verfahren wird das Jodoform durch unser operatives Vorgehen in seiner Wirksamkeit unterstïtat, und wir dürfen demgemäss auch auf sicherere und schnellere Heilung rechnen. Hauptbedingung bleibt hierbei allerdings, dass die anatomischen Verhältnisse es uns als wahrseheinlich erscheinen lassen, den eigentlichen Krankheitsherden beizukommen. Infolgedessen ist leider, wie vorher auseinandergesetzt, ein grosser Theil der sogenannten Senkungsabscesse nach unseren Erfahrungen von dieser Behandlungsart auszuschliessen.

Für die Gelenkerkrankungen jedoch ist diese Methode ausserordentlich empfehlenswerth. Von 28 Gelenkerkrankungen (obne Abscedirung; 19 ostaler Form, 9 Kapselerkrankungen), welche von uns nach Billrotb's Verfahren behandelt worden sind, sind 21 definitiv geheilt, 5 haben Recidive bekommen, ein Patient ist nicht auffindbar, und einer ist bereits 3 Monate nach der Entlassung, jedoch ohne Recidiv, gestorben. In 16 dieser Fälle ist die Primärnaht, in 12 die Secundärnaht angelegt worden. Bei Anlegung der Secundärnaht verfabren wir derart, dass wir nach Beendigung der Operation die Wunde mit Jodoformgaze ausstopfen und alsdann die Wundränder in genauer Naht bis auf eine etwa $2 \mathrm{Cm}$. lange Partie vereinigen. Durch die bestehen bleibende Oeffnung werden die Enden der tamponirenden Gaze herausgefiuhrt und durch die Wundränder dieser Oeffnung 2 bis 3 Fäden gelegt, welche jedoch noch nicht geknüpft werden. Verband. 
Nach 2 Tagen wird der Verband gewechselt, die Gaze entfernt, die Wundhöhle mit Jodoform-Glycerin angefüllt und die bei der ersten Operation gelegten Fäden geknüpft. Bei dieser Art der Secundärnaht wird die zweite Narkose erspart, da das Knüpfen der vorher in Narkose bereits gelegten Fäden von kaum erwähnenswerthen Schmerzäusserungen Seitens der Patienten begleitet ist. Als Vortheil der Secundärnaht ist der Umstand anzuführen, dass wir hierdurch in die Lage versetzt werden, das Jodoform-Glycerin auf eine nicht mehr blutende Wundfläche zu appliciren. Und wenn wir auch nur in wenigen Fällen die von Bill roth erwähnte zu starke Spannung durch nach. folgende Blutung und bierdurch bedingte Störung der prima intentio beobachtet haben, so schützen wir doch durch dieses Verfahren die Wunde vor einem grösseren todten Blutcoagulum, welches nach Ansicht Bramann's als ein besonders günstiger Nährboden für das Tuberkelvirus anzusehen ist.

In den definitiven Resultaten haben wir jedoch, wie ich ausdrücklich bemerken will, keine nennenswerthen Unterschiede zwischen den mit Primär- und den mit Secundärnaht behandelten Fällen constatiren können.

Bei dem Operationsverfahren bei Kapseltuberculose, die noch nicht $\mathrm{zu}$ weit vorgeschritten ist, suchen wir uns die Gelenkhöhle unter möglichster Schonung des Bandapparats zugänglich zu machen und entfernen die erkrankte Kapsel nach Möglichkeit mit Messer, Scheere und scharfem Löffel. In zwei Fällen von Kapseltuberculose des Kniegelenks (Fall 75, 76) bedienten wir uns zweier Längsschnitte, seitlich zur Patella. In dem einen Falle (Fall 75), der vor 2 Jahren operirt worden ist, und den ich jetzt wiederzusehen Gelegenbeit hatte, ist vollkommene Heilung mit völlig freier Beweglichkeit erzielt worden.

In zwei anderen Fällen von Hydrops tuberculosus genu (Fall 79 u. 80) wurde nur ein Längsschnitt, seitlich zur Patella, geführt; man liess die hydropische Fltissigkeit ab und kratzte die Kapsel mit dem scharfen Löffel aus. In beiden Fällen trat völlige Heilung ein, bei dem einen (Fall 79), den ich vor Kurzem untersuchen konnte, mit voller freier Beweglichkeit.

Bei der Kapselerkrankung des Ellbogengelenks bedienten wir uns in zwei Fällen (Fall 77, 78) folgenden Verfahrens. Es wurde ein Längsschnitt am inneren Rande des Olecranon geführt und dieses alsdann an der Basis durch einen Meisselschlag von der Ulna derartig gelöst, dass es noch mit dem Periost in Verbindung blieb; es gelang so nach Einsetzen eines Hakens in das losgelöste Olekranon, 
durch Zug die Gelenkhöhle freizulegen, so dass wir bequem die erkrankten Kapseltheile entfernen konnten. Das Olekranon wurde alsdann durch einige Silbernähte an die Ulna fixirt und der Arm zunächst in Extension verbunden. Wir haben in beiden Fällen definitive Heilung, wenngleich nicht volle freie Beweglichkeit erzielt.

Bei Kapselerkrankungen des Fussgelenks jugendlicher Individuen empfiehlt sich die von Girard angegebene Methode. Man fuhrt einen etwa $3 \mathrm{Cm}$. grossen Längsschnitt an dem vorderen Rande des Malleolus ext. und durchmeisselt ihn an der Stelle, wo er in die Fibula übergeht. Zieht man nun den Malleolus, der durch sein Periost noch in Verbindung mit den benachbarten Weichtheilen geblieben ist, mit einem Haken nach aussen, so erlangt man einen bequemen Zugang in das Gelenk. Ist dann, wie in unserem Falle (Fall 44), eine Entfernung des Talus erforderlich, so hat man genügend Platz, um dieses auszufuhren. Der temporär resecirte Malleolus wird durch einige Periostnähte an die Fibula fixirt. Das von uns so operirte Kind hat nach Mittheilung der Eltern ein bewegliches Fussgelenk.

In all' den anderen Fällen, in denen es sich um die ostale Form der Tuberculose handelt, bedienen wir uns gewöhnlich der typischen Schnittführung, operiren jedoch oftmals alsdann atypisch, indem wir nur das Kranke allein zu entfernen bestrebt sind.

In jenen Fällen von schwerer Gelenktuberculose, welche mit starker Abscedirung und Fistelbildung einhergehen, in jenen Fällen mit tiefliegenden, periarticulären Abscessen, wo die Wahrscheinlichkeit vorliegt, dass trotz sorgsamster Ausräumung doch noch Krankheitsherde übersehen werden, verzichten wir völlig auf die prima intentio und behandeln mit ständiger Jodoform-Glycerinausstopfung.

Wir verfabren hierbei derart, dass wir sterilisirte Gazestreifen mit der Jodoform-Glycerinmischung tränken und mit der so hergestellten Jodoform-Glyceringaze die Ausstopfung bewerkstelligen; bei jedem Verbandwechsel, welcher etwa jeden 5. Tag vorgenommen wird, erfolgt Erneuerung der Ausstopfung. Dieses Verfahren beansprucht allerdings viel Geduld, sowohl von Seiten des Patienten, wie des Arztes. Allein die Resultate, welche wir hiermit erzielt haben - und es handelte sich zumeist um schwere Fälle - sind ausserordentlich befriedigend. Die consequent durchgeführte Tamponade nach Resectionen ist von Kraske bereits im Jahre 1885 auf der Naturforscherversammlung in Strassburg empfohlen worden. Mit Recht bezeichnet Kraske die ständige Tamponade geradezu als antituberculös, einmal weil durch den formativen Reiz des Fremdkörpers eine lebhaftere Gewebsbildung angeregt wird, dann aber auch, weil wir durch diese 
Methode in die Lage versetzt werden, antituberculöse Mittel, so das Jodoform, genügend lange mit verdächtigem Gewebe in Beruhrung zu bringen. Als grosser Uebelstand dieses Verfahrens wird allgemein die Schmerzhaftigkeit und Blutung bei Entfernung der Tampons angesehen, und dieses veranlasste $\mathrm{Kr}$ a sk e, resorbirbares Material, das in die Wunde eingenäht wird, zur Tamponade zu verwenden. Auf diesem Wege die oben erwähnten Uebelstände der Tamponade zu beseitigen, halten wir nicht für zweckmässig, weil wir ja bei diesem von Kraske vorgeschlagenen Verfahren der Hauptvortheile der Tamponade verlustig gehen, der Vortheile, welche darin bestehen, dass wir durch ständige Erneuerung der Tampons immer von Neuem mit dem Jodoform auf die Gewebe einwirken können, sowie dass die nach Bearbeitung mit Löffel und Paquelin sich abstossenden Gewebspartien aus der Wundböhle ausgestossen werden können. Bei der von uns bingegen gelibten consequenten Ausstopfung mit Jodoform-Glyceringaze geniessen wir die Vortheile der Tamponade, ohne ihre Nachtheile zu verspüren. Das Glycerin verhindert nämlich eine Verklebung der Gazestreifen mit den Granulationen, und bei Erneuerung der Tampons bleibt den Patienten Schmerz wie Blutverlust erspart.

Stellen wir nun zum Schluss nochmals auf Grund der angefigten 87 Fälle in aller Kürze die Gesichtspunkte zusammen, von welchen aus wir die Behandlung mit Jodoform-Glycerin empfehlen können.

Fur das Billroth'sche Verfahren eignen sich die Fälle, in denen wir die Wahrscheinlichkeit haben, durch operatives Vorgehen alle Krankheitsherde zu entfernen.

Für die Behandlung mit Punction und Injection von JodoformGlycerin, ein Verfahren, welches den grossen Vortheil hat, dass die Patienten nicht bettlägerig werden, eignen sich die Kapseltuberculosen, sofern sie nicht zu weit vorgeschritten sind, sowie die kalten Abscesse, deren Ausgangspunkte wir entweder nicht kennen oder nicht erreichen können.

Fur die Behandlung mit consequenter Tamponade mit JodoformGlyeeringaze eignen sich einmal die kalten Abscesse, bei denen trotz wiederholter Punction und Injection keine Besserung erzielt worden ist, sowie die, bei welchen das Punctionsverfahren zwar an sich am Platze wäre, sich aber wegen zu starker Spannung und Verdünnung der Haut von selbst verbietet, dann aber besonders alle die schweren Fälle von Gelenktuberculose mit Abscedirung und Fistelbildung, sowie tiberhaupt alle die Fälle, bei denen wir durch die anatomischen Verhältnisse, so besonders bei den Hand- und Fussgelenken, verhindert sind, alle Krankheitsherde zu entfernen. 
Behandl. tuberc. Knochen- u. Gelenkerkrankungen mit Jodoform-Glycerin. 595

Wir verwenden das Glycerin als Vehikel für das Jodoform, weil wir einmal in dieser Form uns leicht ein sterilisirtes Jodoform verschaffen können, dann weil es uns hierdurch ermöglicht wird, das suspendirte Jodoform in alle Tiefen und Taschen der Wunde gelangen zu lassen, ohne dass seine Anwendung, wie beim Aether, von Schmerzen begleitet ist, noch Intoxicationsgefahren zur Folge haben kann, und schliesslich weil das Glycerin die getränkten Gazestreifen an einer Verklebung mit der Wundfläche hindert, wodurch allein eine consequente Tamponade in bequemer Weise ausfubrbar ist.

Von Seiten des Jodoforms haben wir niemals - und wir haben bis $60,0 \mathrm{Grm}$. der 10 proc. Glycerinmischung verwandt - irgend welche Intoxicationserscheinungen beobachtet, niemals Albuminurie, noch Hämoglobinurie.

I. Auguste Grauss, 26 Jahre alt. Kalter Abscess oberhalb des Ligam. Poupartii; Schmerzhaftigkeit an der Synchondrosis sacro-iliaca. 24. April bis 28. Mai 1890. Zweimalige Punction und Injection; Abscess bedeutend verkleinert; gebessert, auf Wunsch entlassen. Juli 1890: Abscess ist gänzlich geschwunden. Gegenwärtiger Zustand: Völlige Heilung. Patientin arbeitet wie früher. Briefliche Mittheilung vom 21. Juni 1892.

2. Sophie Mischberger, 32 Jahre alt. Kalte Abscesse oberhalb des Ligam. Poupartii, in den Adductoren und unterhalb des M. glutaeus; Synchondrosis sacro-iliaca schmerzhaft. Viermalige Punction und Injection. 25. Mai bis 7. August 1891. Patientin wird geheilt entlassen. Nach 5 Monaten noch Jod im Urin nachweisbar. Befund am 18. Juni 1892: Völlige Heilung.

3. Eugen $\mathrm{Kuhn}$, 45 Jahre alt. Kalter Abscess in der linken Kniekehle (Kniegelenk frei) und auf dem Rücken. 25. December 1891 bis 1. Februar 1892. Zweimalige Punction mit nachfolgender Injection; geheilt entlassen. Gegenwärtiger Zustand: Geheilt. Briefliche Mittheilung vom 1. Juli 1892.

4. Melanie Laurent, 34 Jahre alt. Kalter Abscess unterhalb des Ligam. Poupartii; Kyphose in der Lendenwirbelsäule. 6. October bis 8. November 1890. Zweimalige Punction mit nachfolgender Injection; geheilt entlassen. Wiedereintritt am 22. December 1890 mit Abscess. Incision; Primärnaht; geheilt entlassen. Gegenwärtiger Zustand: Geheilt; Abscess ist nicht wieder aufgetreten. Briefliche Mittheilung vom 21. Juni 1892.

5. Karl Sütterlin, 32 Jahre alt. Kalter Abscess unterhalb der Crista ossis ilei. Schmerzhaftigkeit der Spina inf.; Lungenspitzenkatarrh; 16. December 1891 bis 28. Februar 1892. Zweimalige Punction mit nachfolgender Injection. Da keine Verkleinerung eintritt, Incision, Secundärnaht; Sequester an der Spina inf. Geheilt entlassen. Gegenwärtiger Zustand: In der alten Narbe mehrere Fisteln; briefliche Mittheilung vom 20. Juni 1892. 
6. Therese Denis, 34 Jahre alt. Kalter Abscess oberhalb des linken Ligam. Poupartii nach vorangegangener Schmerzhaftigkeit in der Lendenwirbelsäule. 14. April bis 6. Juli 1890. Punction mit Pottin vergeb. lich, da Abscessinhalt zu dickftissig. Incision, Primärnaht; am 14. Tage feine Fistelöffnung in der Narbe; da Abscessinhalt wieder reichlich vorhanden, noclimalige Incision und Primärnaht; dieselbe schneidet durch; Behandlung mit ständiger Ausstopfung mit Jodoform-Glyceringaze. Geheilt entlassen. Gegenwärtiger Zustand: Völlige Heilung; briefliche Mittheilung vom 21. Juni 1892.

7. Franziska Friedmann, 22 Jahre alt. Kalter Abscess am Darmbeinkamm; 15. bis 28. September 1890. Incision; Rauhigkeit am Beckenkamm; Primärnaht. Gelıeilt entlassen. Gegenwärtiger Zustand: Fistel; briefliche Mittheilung vom 20. Juni 1892.

8. Marianne Vorburge r, 61 Jahre alt. Kalter Abscess in der rechten Regio lumbalis. 7. November bis 14. December 1890. Incision; Primärnaht. Geheilt entlassen. Gegenwärtiger Zustand: Fistel, starke Secretion. Briefliche Mittheilung vom 23. Juni 1892.

9. Xaver Martzluff, 4 Jahre alt. Kalter Abscess am Kniegelenk ohne Betheiligung desselben. 11. bis 31. Juli 1890. Incision; Primärnaht; geheilt. Gegenwärtiger Zustand: Fistel; briefliche Mittheilung vom 26. Juni 1892.

10. Josephine Wagner, 5 Jahre alt. Kalter Abscess unterhalb des Ligam. Poupartii. 26. August bis 15. September 1890. Incision; Primärnaht; geheilt. Gegenwärtiger Zustand: Patientin nicht auffindbar.

11. Richard $\mathrm{B}$ a u e r, 13 Jahre alt. Senkungsabscess am Rücken. 5. Mai bis 22. Juni 1890. Primärnaht. Mit Fistel entlassen. Gegenwärtiger Zustand: Fistel; briefliche Mittheilung vom 10. Juli 1892.

12. Johann Dol1, 21 Jahre alt. Kalter Abscess am Trochanter. 11. Juni bis 7. Juli 1891. Incision; Secundärnaht; kein Knochenherd nachweisbar. Geheilt entlassen. Befund vom 1. Juli 1892: Völlige Heilung; Doll ist zum Militär ausgehoben.

13. Christian Spraul, 29 Jahre alt. Kalter Abscess an der rechten Regio lumbalis; Spondylitis lumbalis. 18. Angust bis 21. September 1891. Incision; Secundärnaht. Geheilt entlassen. Gegenwärtiger Zustand: Fistel; briefliche Mittheilung vom 23. Juni 1892.

14. Catharina B o gerer, 65 Jahre alt. Kalter Abscess am Rücken; Kyphose der Brustwirbel. 3. December 1890 bis 18. Januar 1891. Incision; Secundärnaht. Geheilt entlassen. Gegenwärtiger Zustand: Todt.

15. Emil B a rth, 6 Jahre alt. Kalter Abscess oberhalb des Tro. chanter major, stark gespannt. Incision, Rauhigkeit am hinteren Pfannenrand. Primärnaht; dieselbe schneidet durch; darauf ständige Ausstopfung mit Jodoform-Glyceringaze. Geheilt entlassen am 26. April 1891. Recht. winklige Ankylose im Hüftgelenk durch Resection beseitigt; kein Herd, weder in Pfanne noch Kopf, nachweisbar. Gegenwärtiger Zustand: Patient ist vollkommen gesund; briefliche Mittheilung vom 5. Mai 1892.

16. Bruno Herkner, 21 Jahre alt. Kalter Abscess oberbalb des Ligam. Poupartii; Schmerzhaftigkeit in der Lendenwirbelsäule. Lungen- 
Behandl. tuberc. Knochen- u. Gelenkerkrankungen mit Jodoform-Glycerin. 597

spitzenkatarrh; vor Jahresfrist Castration wegen Tuberculose. 18. Februar bis 18. März 1891. Incision; Primärnaht; dieselbe schneidet am 3. Tage durch; ständige Ausstopfung mit Jodoform-Glyceringaze. Mit Fistel entlassen. Befund am 21. Juni 1892: Die Fistel hat sich geschlossen; völlige Heilung.

17. Caroline Treiber, 24 Jahre alt. Kalter Abscess in der linken Lendengegend; Haut geröthet. 4. November 1890 bis 17. Januar 1891. Incision; sehr grosse Höhle; Secundärnaht. Da sie am 2. Tage durchgeschnitten hat, ständige Ausstopfung mit Jodoform-Glyceringaze. Patientin wird mit kleiner Fistel auf Wunsch entlassen. Gegenwärtiger Zustand: Patientin nicht auffindbar.

18. Carl Tochter, 4 Jahre alt. Kalter Abscess, von Kyphose der Lendenwirbel ausgehend. 5. September 1890 bis 21. März 1891. Sehr starke Spannung. Incision mit Secundärnaht, welche bei der sehr verdunnten Haut am 2. Tage durchschneidet. Daranf ständige Ausstopfung mit Jodoform-Glyceringaze. Patient mit Fistel entlassen. Gegenwärtiger Zustand: Fistel; briefliche Mittheilung vom 8. Mai 1892.

19. Mathilde Kessler, 10 Jahre alt. Kalte Abscesse oberhalb beider Ligam. Poupartii. Kyphose. 8. Januar bis 10. Mai 1891. Incision; Secundärnaht; da diese durchschneidet, ständjge Ausstopfung mit Jodoform-Glyceringaze. Geheilt entlassen; später Exitus an Miliartuberculose.

20. Matthias Sch uitterle, 39 Jahre alt. Kalter Abscess in der Regio lumbalis; Spondylitis lumbalis. 5. Januar bis 17. Februar 1891. Ständige Ausstopfung mit Jodoform-Glyceringaze, da die Haut sich nach der Incision zu stark retrahirt. Geheilt entlassen. Befund vom 20. Juni 1892: Völlige Heilung; nirgends Schmerzhaftigkeit noch Abscessbildung.

21. Marie Georg, 67 Jahre alt. Kalter Abscess in der Regio lum balis; Fistel. 30. Januar bis 15. März 1891. Incision; Resection der 7. und 8. Rippe; ständige Ausstopfung. Ungeheilt entlassen.

22. Ludwig $\mathrm{W}$ arth, 10 Jahre alt. Rippencaries. 22. September bis 11. October 1890. Rippenresection; Anfüllung mit Jodoform-Glycerin; Primärnaht. Geheilt entlassen. Gegenwärtiger Zustand: Fistel mit mässiger Secretion. Briefliche Mittheilung vom 2. Mai 1892.

23. Magdalena Bräutiga m, 54 Jahre alt. Rippencaries. 16. Februar bis 1. Juli 1891. Incision; Resection der 7. und 8. Rippe; ständige Ausstopfung mit Jodoform-Glycerin. Ungeheilt entlassen.

24. August M üller, 61 Jahre alt. Rippencaries. 20. Mai bis 7. Juli 1891. Resection; ständige Ausstopfung mit Jodoform-Glycerin. Geheilt entlassen. Gegenwärtiger Zustand: Geheilt. Briefliche Mittheilung vom 5. Juli 1892.

25. Georg M üller, 51 Jahre alt. Rippencaries. 30. Juni bis 9. August 1891. Resection; ständige Ausstopfung mit Jodoform-Glycerin. Geheilt entlassen. Patient ist 2 Monate später an Phthisis pulmonum gestorben. Die Operationswunde war völlig geheilt.

26. Michael Ko ebel, 40 Jahre alt. Caries manubrii sterni. 7. Januar bis 11. Februar 1892. Resection; ständige Ausstopfung mit Jodoform-Glycerin. In Heilung entlassen. Gegenwärtiger Zustand: Geheilt; 
keine Fistel, keine Schmerzhaftigkeit. Briefliche Mittheilung vom 5. Juli 1892.

27. Emil Scherr, 10 Jahre alt. Herd im Condyl. int. humeri, ohne Betheiligung des Gelenks. 2. März bis 9. April 1891. Incision, Auskratzen; Anftullung mit Jodoform-Glycerin; Primärnaht, welche am 2. Tage durchschneidet. Ständige Ausstopfung mit Jodoform-Glycerin. Geheilt entlassen. Gegenwärtiger Zustand: Geheilt. Briefliche Mittheilung vom 2. Mai 1892.

28. Theodor Fretz, 35 Jahre alt. Herd im Trochanter major. 16. Juni bis 2. September 1891. Incision, Aufmeisselung; ständige Ausstopfung mit Jodoform.Glycerin. Geheilt entlassen. Gegenwärtiger Befund: Geheilt. Briefliche Mittheilung vom 2. Juli 1892.

29. Anton II att, 15 Jahre alt. Herd im Malleol. int. 4. November 1891 bis 17. Januar 1892. Incision, Aufmeisselung; ständige Ausstopfung mit Jodoform-Glycerin. Geheilt entlassen. Gegenwärtiger Zustand: Geheilt. Briefliche Mittheilung vom 4. Juli 1892.

30. Emil K opp, 10 Jahre alt. Gonitis tuberc. 5. Juli bis 7. August 1890. Resection; Jodoform-Glycerin, Primärnaht. Geheilt entlassen. Gegenwärtiger Zustand: Geheilt. Briefliche Mittheilung vom 10. Juli 1892.

31. Eduard Frits ch, 5 Jahre alt. Gonitis tuberc. 21. Juli bis 7. August 1890. Jodoform-Glycerin; Resection; Primärnaht; geheilt entlassen. Gegenwärtiger Zustand: Recidiv; briefliche Mittheilung vom 11. Juli 1892.

32. Marie Kittel, 10 Jahre alt. Gonitis tuberc. 1. November 1891 bis 15. Februar 1891. Resection. Jodoform-Glycerin; Primärnaht; geheilt entlassen. Gegenwärtiger Zustand: Recidiv; briefliche Mittheilung vom 3. Mai 1892.

33. Nikolaus Bernhard, 35 Jahre alt. Gonitis tuberc. 1. December 1890 bis 16. April 1891. Resection; Jodoform-Glycerin; Primärnaht. Geheilt entlassen. Gegenwärtiger Zustand: Patient geht ohne Schmerzen, kleine Fistel in der Narbe. Briefliche Mittheilung rom 23. Juni 1892.

34. Engenie Fischer, 7 Jahre alt. Tuberculose des Fussgelenkes. 6. bis 27. August 1890. Typische Resection; Entfernung des Talus; Jodoform-Glycerin; Primärnaht. Geheilt entlassen. Befund vom 1. Juni 1892: Geheilt; Ankylose.

35. Jacob Schmidt, 5 Jahre alt. Coxitis tuberc. 24. October bis 12. December 1890. Resection; Jodoform-Glycerin; Secundärnaht. Geheilt entlassen. Patient ist nach brieflicher Mittheilung 3 Monate später an Miliartuberculose gestorben. Das Hüftgelenk war vollkommen ausgeheilt; Patient konnte gehen.

36. Florentine Bucher, 8 Jahre alt. Coxitis tuberc. 13. Februar bis 17. März 1891. Resection; Anfüllung mit Jodoform.Glycerin; Secnndärnaht; geheilt entlassen. Gegenwärtiger Zustand: Vollkommen ausgeheilt nach brieflicher Mittheilung vom 10. Mai 1892.

37. Eugenie Wagner, 3 Jahre alt. Coxitis tuberc. 25. Februar bis 22. März 1891. Resection; Anfüllung mit Jodoform-Glycerin; Secundärnaht; geheilt entlassen. Gegenwärtiger Zustand: Vollkommen ausgeheilt nach brieflicher Mittheilung vom 9. Mai 1892. 
38. Margarethe Vatel, 16 Jahre alt. Tuberculose des Fussgelenks. 19. December 1890 bis 21. April 1891. Resection; Anfüllung mit Jodoform-Glycerin; Secundärnaht; geheilt entlassen. Gegenwärtiger Zustand: Geheilt; briefliche Mittheilung vom 24. Juni 1892.

39. Ida $\mathrm{H} \mathrm{u} \mathrm{ck}, 36$ Jahre alt. Tuberculose des Ellbogengelenks. 4. Februar bis 10. März 1891. Resection; Jodoform-Glycerin; Secundärnaht; geheilt entlassen. Gegenwärtiger Zustand: Geheilt; Patientin näht wieder. Briefliche Mittheilung vom 23. Juni 1892.

40. Marie Lutz, 55 Jahre alt. Fremdkörper-Gelenksentzlindung des Ellbogengelenks. 23. Februar bis 10. März 1891. Arthrektomie; Primärnaht. Wiedereintritt am 8. Mai. Typische Tuberculose des Ellbogengelenks. Resection; Jodoform-Glycerin; Secundärnaht. Am 1. Juli geheilt entlassen. Befund am 16. Juli 1892: Vollkommene Heilung, völlig freie Beweglichkeit, auch Pronation und Supination.

41. Marie Meyer, 19 Jahre alt. Gonitis tuberc. 20. Mai bis 20. Juni 1891. Resection; Jodoform-Glycerin; Secundärnaht; geheilt entlassen. Gegenwärtiger Zustand: Geheilt; keine Fistel, keine Schmerzen. Briefliche Mittheilung vom 22. Juni 1892.

42. Eugenie Stahl, 25 Jahre alt. Gonitis tuberc. 20. Juni bis 19. Juli 1891. Resection; Jodoform-Glycerin; Secundärnaht; geheilt entlassen. Gegenwärtiger Zustand: Völlige Heilung. Briefliche Mittheilung vom 23. Juni 1892 .

43. Emma Wanz, 24 Jahre alt. Kapseltuberculose im Kniegelenk. 8. December 1890 bis 14. Februar 1891. Arthrektomie; Jodoform-Glycerin; Primärnaht; geheilt entlassen. Wiedereintritt am 4. Januar 1892. Starke Schmerzhaftigkeit; Resection wegen Herde in den Condylen. JodoformGlycerin; Secundärnaht; geheilt entlassen. Gegenwärtiger Zustand: Geheilt; keine Schmerzhaftigkeit. Briefliche Mittheilung vom 23. Juni 1892.

44. Therese Higel, 4 Jahre alt. Tuberculose im Fussgelenk. 16. Februar bis 15. März 1891. Temporäre Resection des Malleolus ext. nach Girard. Entfernung des Talus; Jodoform-Glycerin; Secundärnaht; geheilt entlassen. Gegenwärtiger Zustand: Völlig geheilt; ganz geringe Verkuirzung; bewegliches Fussgelenk; Kind läuft gut. Briefliche Nachricht vom 25. Juni 1892.

45. Barbara Brobecker, 10 Jahre alt. Gonitis tuberc. 13. Mai bis 10. Juni 1890. Resection; Jodoform-Glycerin; Primärnaht; geheilt entlassen. Gegenwärtiger Zustand: Völlig geheilt; „springt umher"; briefliche Mittheilung vom 10. Juli 1892.

46. Magdalena Schmied, 8 Jahre alt. Tuberculose des Fussgelenks. 16. Mai bis 17. Juli 1890. Resection; Jodoform-Glycerin; Primärnaht; geheilt entlassen. Gegenwärtiger Zustand: Völlige Heilung; briefliche Mittheilung vom 12. Juli 1892.

47. Katharina Kössler, 12 Jahre alt. Gonitis tuberc. 12. Juni bis 18. Juli 1890. Resection; Jodoform-Glycerin; Primärnaht; geheilt entlassen. Gegenwärtiger Zustand: Geheilt. Briefliche Mittheilung vom 12. Juli 1892 . 
48. Emil Fonné, 22 Jahre alt. Tuberculose des Handgelenks. 20. Mai bis 25. Juli 1891. Dorsalschnitt; Entfernung der Handwurzelknochen; ständige Ausstopfung mit Jodoform-Glycerin. Geheilt entlassen; geringe Beweglichkeit der Hand. Gegenwärtiger Zustand: Geheilt; steifes Handgelenk. Briefliche Mittheilung vom 2. Juli 1892.

49. Constantin G érard, 28 Jahre alt. Tuberculose des Handgelenks und der Sehnenscheiden. 14. Mai bis 14. Juli 1891. Resection; Auslöffelung; ständige Ausstopfung mit Jodoform-Glycerin; ungeheilt entlassen.

50. Henriette Riss, 44 Jahre alt. Tuberculose des Handgelenks. 23. Februar bis 2. Mai 1891. Dorsalschnitt; Auslöffelung der Handwurzelknochen; ständige Ausstopfung mit Jodoform-Glycerin; geheilt entlassen. Gegenwärtiger Zustand: Todt.

51. August Lisch ka, 16 Jahre alt. Tuberculose des Ellbogengelenks. 15. August bis 1. November 1891. Resection; ständige Ausstopfung mit Jodoform-Glycerin; geheilt entlassen. Befund am 1. Juni 1892: Geheilt, völlige Ankylose; kein Recidiv.

52. Barbara Renner, 49 Jahre alt. Tuberculose des Ellbogengelenks. 15. April bis 20. Juni 1891. Resection; ständige Ausstopfung mit Jodoform-Glycerin; geheilt entlassen. Gegenwärtiger Zustand: Reeidiv; briefliche Mittheilung vom 18. Juli 1892.

53. Peter $\mathrm{Re} e \mathrm{~b}, 38 \mathrm{Jahre}$ alt. Coxitis mit Abscessbildung. 20. Juni bis 20. August 1891. Resection; ständige Ausstopfung mit Jodoform-Glycerin; geheilt entlassen. Gegenwärtiger Zustand: Geheilt; keine Fistel, keine Schmerzhaftïgkeit. Briefliche Mittheilung vom 3. Juli 1892.

54. Emil Origas, 8 Jahre alt. Coxitis mit grossen Abscessen und Fistel; Pfannenperforation. 25. Juli bis 29. December. Resection; ständige Ausstopfung mit Jodoform-Glycerin. Patient wird ungeheilt auf Wunsch entlassen.

55. Eugen Schaal. Coxitis mit grossen Abscessen und Fistel. 21. Januar bis 5. April 1891. Resection; ständige Ausstopfung mit Jodoform-Glycerin. Patient geheilt entlassen. Gegenwärtiger Zustand: Geheilt, keine Schmerzhaftigkeit; Patient geht in die Schule. Briefliche Mittheilung vom 3. Mai 1892.

56. Elisabeth $\mathrm{Sch}$., 9 Jahre alt. Coxitis mit Abscess. 5. Juli bis 10. August 1890. Resection; Ausstopfung mit Jodoform-Glycerin; geheilt. Gegenwärtiger Zustand: Vollkommene Heilung; Hüftgelenk beweglich.

57. Theobald Weiss, 10 Jahre alt. Coxitis mit grossen Abscessen. 7. Januar 1891 bis 27. Februar 1892. Resection und ständige Ausstopfung mit Jodoform-Glycerin. Die Wunde ist fast völlig geheilt; Exitus an Basilarmeningitis.

58. Wilhelmine Vetter, 6 Jahre alt. Coxitis mit Abscessen. 4. März bis 24. Mai 1891. Resection; ständige Ausstopfung mit Jodoform-Glycerin. Geheilt entlassen. Gegenwärtiger Zustand: Patientin ist kurze Zeit nach ihrer Entlassung an Miliartuberculose gestorben.

59. Emil Lenderer, 10 Jahre alt. Coxitis mit Abscess und Fisteln. 30. Mai bis 21. August 1890. Resection; ständige Ausstopfung mit Jodo- 
Behandl, tuberc. Knochen- u. Gelenkerkrankungen mit Jodoform-Glycerin. 601

form-Glycerin; geheilt entlassen. Gegenwärtiger Zustand: Recidiv; briefliche Mittheilung vom 12. Juli 1892.

60. Luise Verflamme, 27 Jahre alt. Tuberculöse Entzindung des Kniegelenks mit Abscessen und Fisteln. 20. Februar bis 3. Juni 1891. Resection; ständige Ausstopfung mit Jodoform-Glycerin. Geheilt entlassen. Befund am 25. Juni 1892: Völlige Heilung; Ankylose.

61. Marie Eckerdt, 26 Jahre alt. Gonitis tuberc. mit Fisteln. 10. August bis 15. November 1891. Resection; ständige Ausstopfung mit Jodoform-Glycerin. Geheilt entlassen. Gegenwärtiger Zustand: Patientin ist nicht auffindbar.

62. Paul Motk, 17 Jahre alt. Tuberculose des Fussgelenks. 25. Juni bis 13. September 1891. Resection; ständige Ausstopfung mit JodoformGlycerin. Geheilt entlassen. Wiedereintritt am 5. Januar 1892. Recidiv; Betheiligung des Metatarsus. Nachresection; Ausstopfung; trotz ständiger Ausstopfung mit Jodoform-Glycerin keine Besserung, schliesslich Amputatio.

63. Ludwig Hochstetter, 30 Jahre alt. Tuberculose des Fussgelenks mit Abscedirung. 25. August bis 21. September 1891. Resection; ständige Ausstopfung mit Jodoform-Glycerin. Geheilt entlassen. Gegenwärtiger Zustand: Geheilt; Ankylose. Briefliche Mittheilung vom 4. Juli 1892.

64. Georg Wendlin, 40 Jahre alt. 'Tuberculose des Fussgelenks mit Abscessbildung. 29. Juni bis 4. November 1891. Resection; ständige Ausstopfung mit Jodoform-Glycerin. Da keine deutliche Besserung, schliesslich Amputatio femoris, indem gleichzeitig Gonitis tuberc. bestand.

65. Katharina Jean nout, 32 Jahre alt. Fussgelenktuberculose (sehr schwerer Fall, zur Amputation geschickt). 2. Mai bis 9. Juli 1891. Resection; ständige Ausstopfung mit Jodoform-Glycerin. Geheilt entlassen mit Schienenschuh. Wiedereintritt am 7. Februar 1892. Recidiv; Amputation.

66. Therese Martin, 50 Jahre alt. Fussgelenktuberculose (Amputationsfall). 12. Mai bis 19. Juli 1891. Resection; ständige Ausstopfung mit Jodoform-Glycerin. Geheilt mit Schienensehuh entlassen. 1. Deeember 1891 Recidiv. Patientin verweigert jede Operation.

67. Eugen Lor en z, 10 Jahre alt. Fussgelenktuberculose; starke Zerstörung; Abscessbildung. 9. Juli bis 13. September 1890. Typische Resection; ständige Ausstopfung mit Jodoform-Glycerin. Geheilt entlassen, rechtwinklige Ankylose. Gegenwärtiger Zustand: Geheilt, Patient „springt umher". Briefliche Mittheilung vom 3. Mai 1892.

68. Johann Schiltz, 12 Jahre alt. Fussgelenktuberculose. 6. Juli bis 10. September 1890. Typische Resection; ständige Ausstopfung mit Jodoform-Glycerin. Geheilt entlassen; rechtwinklige Ankylose. Gegenwärtiger Zustand: Am 31. Juli 1891 gestorben. Fussgelenk war geheilt und geschlossen.

69. Luise G o n der, 22 Jahre alt. Herd im Calcaneus, Tuberculose des Metatarsus; Auslöffelung. 4. August 1891 bis 27. März 1892. Ständige Ausstopfung mit Jodoform-Glycerin; wiederholte Nachoperationen; ungeheilt entlassen.

70. Jacob Willm, 63 Jahre alt. Tuberculose des Metatarsus. 16. Febraar bis 28. März 1891. Auslöffelung; ständige Ausstopfung mit Jodo- 
form-Glycerin; geheilt entlassen. Gegenwärtiger Befund: Geheilt; keine Schmerzhaftigkeit. Briefliche Mittheilung vom 3. Juli 1892.

71. Christian Weck, 60 Jahre alt. Tuberculöse Caries der Hand. wurzel. 30. Juni bis 23. August 1891. Auslöffelnng; Ausstopfung mit Jodoform-Glycerin; in Heilung entlassen. Gegenwärtiger Zustand: Patient hat noch eine Fistelöffnung, kann jedoch arbeiten. Briefliche Mittheilung vom 10. Juli 1892.

72. H..., 6 Jahre alt. Caries des Os cuneiforme. 1. Juni bis November 1891. Zuerst dreimalige Injection, danach bedeutende Besserung; keine Schmerzhaftigkeit. Nach 6 Wochen starke Schmerzhaftigkeit; Abscess; Incision; Auslöffelung; ständige Ausstopfung mit Jodoform-Glycerin. Geheilt entlassen. Gegenwärtiger Zustand: Geheilt.

73. Ludwig Grandgeorge, 34 Jahre alt. Kapseltuberculose des Kniegelenks. 12. Februar bis 1. April 1891. Kapselexstirpation; Jodoform-Glycerin; Primärnaht; geheilt entlassen. Gegenwärtiger Zustand: Geheilt; bei längerem Gehen leichte Ermüdung. Briefliche Mittheilung vom 8. Juli 1892 .

74. Carl Hegler, 4 Jahre alt. Kapseltuberculose des Kniegelenks. 20. Juni bis 7. August 1890. Arthrektomie; Jodoform-Glycerin; Primärnaht. Geheilt entlassen. Gegenwärtiger Zustand: Patient ist nicht auffindbar.

75. Joseph $\mathrm{W}$ a g $\mathrm{n}$ er, 4 Jahre alt. Kapseltubereulose des Kniegelenks. 5. Mai bis 2. Juni 1890. Arthrektomie; zwei seitliche Schnitte; JodoformGlycerin; Primärnaht; geheilt entlassen. Befund vom 1. Juni 1892: Völlige Heilung mit freiem Gelenk.

76. Friedrich Böckel, 21 Jahre alt. Kapseltuberculose des Kniegelenks. 26. August bis 13. October 1890. Zwei seitliche Schnitte; Arthrektomie; Jodoform-Glycerin; Primärnaht. Geheilt entlassen. Gegenwärtiger Zustand: Geheilt; briefliche Mittheilung vom 29. Juni 1892.

77. Anna Latzer, 18 Jahre alt. Kapseltuberculose des Ellbogengelenks. 1. Juli bis 1. August 1891. Eröffnung des Gelenks. Temporäre Resection des Olekranon; Arthrektomie; Jodoform-Glycerin; Secundärnaht. Geheilt entlassen. Befund am 1. Juli 1892: Geheilt; keine Schmerzhaftigkeit; Beweglichkeit zwischen $\angle$ von $160-120^{\circ}$. Pronation und Supination nicht ausführbar.

78. Peter Kremer, 43 Jahre alt. Kapseltuberculose des Ellbogengelenks. 12. December 1890 bis 10. Februar 1891. Temporäre Resection des Olekranon; Arthrektomie; Jodoform-Glycerin; Secundärnaht. Geheilt entlassen. Gegenwärtiger Zustand: Geheilt. Briefliche Mittheilung vom 26. Juni 1892.

79. Arthur Schubert, 15 Jahre alt. Hydrops tuberc. des Kniegelenks. 17. December 1891 bis 13. Januar 1892. Ein seitlicher Schnitt. Auskratzen; Jodoform.Glycerin; Primärnaht. Bei der Entlassung besteht keine Schmerzhaftigkeit; Tanzen der Patella. Befund vom 26. Juni 1892 : Keine Schmerzhaftigkeit; völlig freie Beweglichkeit; starke Verdickung an den Gelenkenden.

80. Emil Albasser, 19 Jahre alt. Hydrops tubere. des Kniegelenks. 23. November 1891 bis 19. Januar 1892. Ein seitlicher Schnitt; Auskratzen; Jodoform-Glycerin; Primärnalt; geheilt entlassen. Gegenwärtiger 
Behandl. tuberc. Knochen- u. Gelenkerkrankungen mit Jodoform-Glycerin. 603

Zustand: Vollständig geheilt; Patient kann arbeiten. Briefliche Mittheilung vom 24. Juni 1892.

81. Christine Heidt, 46 Jahre alt. Kapseltuberculose des Fussgelenks. 18. April bis 20. Mai 1891. 3 Jodoform-Glycerininjectionen. Geheilt entlassen. Gegenwärtiger Zustand: Geheilt; bei längerem Gehen leichte Ermüdung. Briefliche Mittheilung vom 11. Juli 1892.

82. Carl Hah n, 18 Monate alt. Kapseltuberculose des Kniegelenks. Spitzwinklige Contractur. .....5 Injectionen in 2 Monaten; letzte Injection am 15. Mai 1891. Beseitigung der Contractur durch 8 tägige Extension. Geheilt entlassen. Befund vom 1. Juli 1892: Vollkommene Heilung; völlig freie Beweglichkeit.

83. Albert Dillinger, 12 Jahre alt. Kapseltuberculose des Handgelenks. .....5 Injectionen innerhalb 7 Wochen; letzte Injection am 30. Mai 1892. Gegenwärtiger Zustand: Vollkommene Heilung; völlig freie Beweglichkeit. Befund vom 20. Juli 1892: Völlige Heilung.

84. Carl M üller, 15 Jahre alt. Kapseltuberculose des Fussgelenks. 5 Injectionen in 7 Wochen; letzte Injection am 4. Juni 1892. Schwellung und Schmerzhaftigkeit völlig geschwunden; Bewegungen etwas beschränkt. Befund rom 20. Juli 1892: Patient geht ohne Schmerzen.

85. Alexander Gỉlling, 15 Jahre alt. Kapseltuberculose des Kniegelenks. . . . . . 5 Injectionen in 6 Wochen; letzte Injection am 21. Mai 1892. Schmerzhaftigkeit und Schwellung völlig geschwunden. Flexion im Knie bis zum $\angle$ von $90^{\circ}$ ausfihrbar. Befund vom 15. Juli 1892: Keine Schmerzhaftigkeit, keine Schwellung.

86. Gertrud $\mathrm{Kuhn}, 6$ Jahre alt. Coxitis; tiefer Abscess oberhalb des Trochanter major. Viermalige Punction und Injection von JodoformGlycerin innerhalb 5 Wochen. Geheilt mit Ankylose, entlassen am 1. Juni 1891. Gegenwärtiger Zustand: Patientin geht ohne Beschwerden umher. Briefliche Mittheilung vom 7. Mai 1892.

87. Emil Bucher, 12 Jahre alt. Coxitis mit mehrfachen Abscessen. 5 malige Punction und Injection von Jodoform.Glycerin innerhalb 7 Wochen. Abscesse füllen sich stets von Neuem; Schmerzhaftigkeit unverändert. Resection (Erkrankung der Pfanne). Ständige Ausstopfung. Geheilt entlassen am 12. August 1891. Befund am 1. Juli 1892: Völlige Heilung, geringe Beweglichkeit. 